(C) 2015 IEEE. Personal use of this material is permitted. Permission from IEEE must be obtained for all other uses, in any current or future media, including reprinting/republishing this material for advertising or promotional purposes, creating new collective works, for resale or redistribution to servers or lists, or reuse of any copyrighted component of this work in other works. 


\title{
A Compact Microstrip Phase Shifter Employing Reconfigurable Defected Microstrip Structure (RDMS) for Phased Array Antennas
}

\author{
Can Ding, Student Member, IEEE, Y. Jay Guo, Fellow, IEEE, \\ Pei-Yuan Qin, Member, IEEE, and Yintang Yang, Member, IEEE
}

\begin{abstract}
A compact phase shifter using reconfigurable defected microstrip structure (RDMS) is proposed for phased array antennas. A complete phase shifter design scheme is described which serves as an engineering guidance. Stepwise phase shifters that have phase-shifting ranges of $90^{\circ}$ and $180^{\circ}$ with a step size of $45^{\circ}$ are designed, fabricated, and tested. The experimental results demonstrate that the size, maximum insertion loss of the phase shifters, and the quantity of the lumped elements are reduced by $80 \%-90 \%, 25 \%-30 \%$, and $50 \%$, respectively, compared to our previous work. Subsequently, a 4-element phased array is built employing such RDMS-based phase shifters, realizing a size reduction of $55 \%$ in the array size. The measurement results show that the phased array antenna is able to switch its main beam between $-15^{\circ}, 0^{\circ}$, and $15^{\circ}$ in the $\mathrm{H}$-plane, with the average realized gain around $10 \mathrm{dBi}$.
\end{abstract}

Index Terms-Reconfigurable defected microstrip structure (RDMS), phase shift, insertion loss, phased array antennas.

\section{INTRODUCTION}

$\mathbf{P}$ HASED array antennas can achieve analogue beamforming $[1,2]$ with high gains and are extensively utilized in satellite communications, radar systems, and other military applications [3]. Usually, a large number of phase shifters are employed in a phased array antenna, and thus their cost, size, and integration method are of great concerns [4]. The most popular phase shifters used in phased array antennas are diode phase shifters [5-8] and ferrite phase shifters [9-11]. Diode phase shifters have fast switching times, low weights, low costs, but high insertion losses [12], whereas ferrite phase shifters are relatively bulky and heavy, and require significant switching power compared with diode phase shifters [13].

Recently, phase shifters based on defected ground structure (DGS) [14] are developed [15-19]. For most of the designs, multi-layer structures and microelectromechanical systems (MEMS) are used. Therefore, they suffer from high cost and

Manuscript received December 19, 2013; Revised June 26, 2014; Accepted February 9, 2015; This work was supported by the National Natural Science Foundation of China under Grant 61172030.

C. Ding is with School of Micro-Electronics, Xidian University, Xi'an, Shaanxi, 710071, China. He is a receipt of a Macquarie University Research Excellence Scholarship and is under a cotutelle arrangement between Macquarie University and Xidian University. He is also with CSIRO DPaS Flagship, Marsfield, NSW, Australia, 2122 (e-mail: dingcantony@163.com).

Y. J. Guo and P. Y. Qin are with Faculty of Engineering and IT, University of Technology Sydney (UTS), Sydney, NSW, Australia, 2122 (e-mail: Jay.Guo@uts.edu.au, Peiyuan.Qin@uts.edu.au).

Y. T. Yang is with the Key Laboratory of Wide Band-Gap Semiconductor Materials and Devices of Ministry of Education, School of MicroElectronics, Xidian University, Xi'an, Shaanxi, 710071, China (e-mail: ytyang@xidian.edu.cn). high fabrication complexity, and may be difficult to integrate in microstrip systems, which substantially limits their applications. As a dual structure of DGS, the defected microstrip structure (DMS) [20] also has the potential to provide phase shift. In [21], C-shaped slots are etched on microstrip lines in the feed network for phase alignment. However, each slot only has a fixed phase delay of $3^{\circ}$ at $12.5 \mathrm{GHz}$. A reconfigurable DMS (RDMS) unit has been proposed by the authors for the first time to design phase shifters [22]. The configuration of a single RDMS unit is shown in Fig. 1(a). It is etched on a microstrip line with a characteristic impedance of $23 \Omega$ and can achieve a phase shift of $17^{\circ}$ at $5.2 \mathrm{GHz}$. Tapered microstrip lines are employed to connect the RDMS unit to the $50 \Omega$ microstrip system. The RDMS based phase shifter has the advantages of low cost, low loss, simple fabrication process, and easy integration with microstrip system. However, it has the disadvantages of large electrical size and high volume of lumped elements that could affect its applications and increase the implementation cost.

In this paper, a new type of RDMS unit with a compact size and a simpler structure is proposed as shown in Fig. 1(b). Based on this RDMS unit, a complete design scheme of stepwise phase shifters consisting of three steps is presented. Each step is supported by simulations and experiments and its mechanism is explained using qualitative equations. Various phase shifters are obtained by following the proposed design scheme. Comparing the obtained phase shifters with the ones in [22], significant reductions in the size, cost and insertion loss are achieved, while the phase-shifting range maintains at the same level. Moreover, the phase shifters are utilized in a 4element phased array to achieve beam steering, which validates the feasibility of the phase shifters and shows substantial size reduction of the array.

The contribution of this paper can be summarized as follows: 1) A new configuration of the RDMS is proposed that leads to significant reductions in size and cost, and an improvement in performance, compared to the designs in [22]; 2) A complete design scheme and optimizing methodology of stepwise phase shifters are presented; 3) This paper provides insights of the phase-shifting mechanism and insertion loss reduction by using equivalent circuits and qualitative equations.

The paper is organized as follows. Following the introduction, the structure and phase shift mechanism of the RDMS unit are described and various RDMS units are presented in Section II. In Section III, RDMS groups are obtained by cascading RDMS units. Then, stepwise phase shifters are 
realized and their advantages are discussed in Section IV. Section V illustrates the RDMS phase shifter's application in a 4-element beam steering antenna array. Finally, the paper is concluded in Section VI.

\section{RDMS UNIT}

\section{A. Structure and phase-shifting mechanism}

The structures of the original RDMS unit [22] and the new one proposed in this paper are given in Fig. 1(a) and 1(b), respectively. As shown in the figure, both RDMS units are built by etching a rectangular slot with a size of $W_{\text {slot }} \times L_{\text {slot }}$ on the microstrip line to introduce a defect. PIN diodes are inserted into the edges of the slot area, which enables structural reconfiguration and results in a reconfigurable DMS (RDMS) unit. Capacitors and metal stubs used for capacitor mounting are placed in the middle of the slot to achieve RF continuity when the diodes are turned off. The dimensions $g_{1}=0.4$ $\mathrm{mm}$ and $g_{2}=0.7 \mathrm{~mm}$ are determined by the mounting requirements of the lumped elements [24,25]. A 1.524- $\mathrm{mm}$ thick Rogers4003 substrate $(\varepsilon=3.55, \delta=0.0027)$ is used in the design.

It is noted that the main differences between two RDMS units shown in Fig. 1 manifested in the width of the microstrip line, the presence of the tapered microstrip lines, and the number of the stubs. We use only one pair of stubs in the new RDMS unit [Fig. 1(b)] rather than two pairs in the original RDMS unit [Fig. 1(a)], so that the width of microstrip line $W$ can be reduced from $10 \mathrm{~mm}$ to $3.4 \mathrm{~mm}$. This way, the tapered microstrip lines used to connect the RDMS unit to the $50 \Omega$ port are eliminated. The phase shift and insertion loss can remain almost the same by tuning the slot dimensions $W_{\text {slot }}$ and $L_{\text {slot }}$.

The phase shift is achieved by controlling the PIN diodes to obtain different current paths. By turning the diodes "on" or "off", the RDMS unit can work in two different states, namely, the "All-on" and "All-off" states, respectively. In the "All-on" state, both the diodes and capacitors allow the currents to go through them with minor losses. Numerical simulations find that the currents concentrate at the edges of the RDMS unit, which is similar to that of a uniform microstrip line. In the "All-off" state, the currents concentrate on the stub since the "off" state diodes can be seen as open circuits. Therefore, phase shift is realized due to the different current path in the two states. It can be deduced that the phase shift $\theta$ is a function of $t$ and $L_{\text {slot }}$ which affect the current path and can be expressed as

$$
\theta=F\left(t, L_{\text {slot }}\right) .
$$

Although the width of the new RDMS unit $W$ in this paper has been significantly reduced (from $10 \mathrm{~mm}$ to $3.4 \mathrm{~mm}$ ), the value of $t\left[t=\left(W_{\text {slot }}-g_{2}\right) / 2\right.$ for the new RDMS unit] can be maintained similar to that of the original one by tuning $W_{\text {slot }}$. Therefore, the width reduction does not degrade the phase shift of the RDMS unit.

Regarding the insertion loss of the RDMS unit, it is very small in the All-on state, since the unit behaves like a uniform

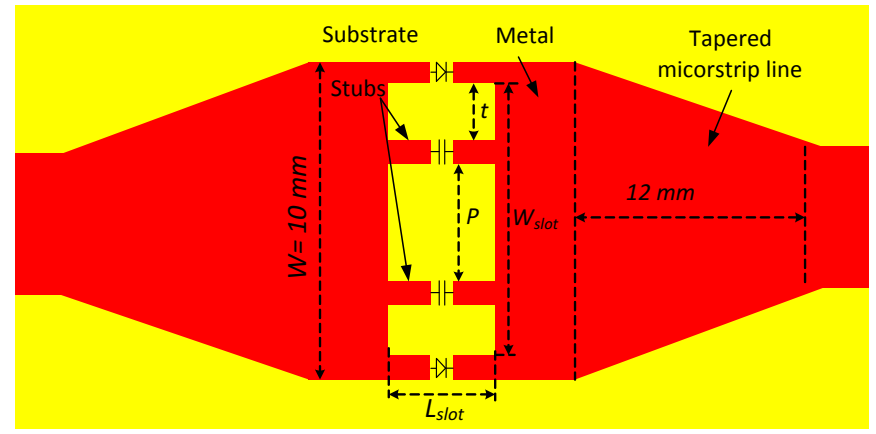

(a)

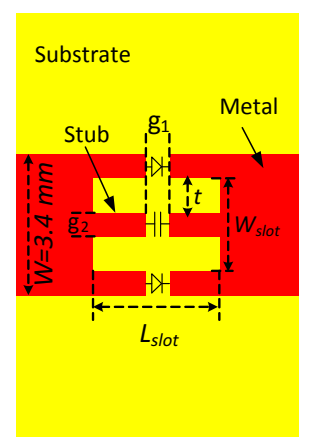

(b)

Fig. 1. Configurations of (a) the original RDMS unit and (b) the new RDMS unit.

microstrip line. It can be expressed by

$$
L_{u}(\text { All-on })=L_{m}+L_{d r},
$$

where $L_{m}$ represents the loss of a conventional microstrip line and $L_{d r}$ represents the sum of the diode loss and radiation loss.

In the All-off state, the RDMS unit introduces noticeable reflection which results in the extra transmission degradation. Here we define the degradation caused by the reflection as $F_{1}=F_{1}\left(W_{\text {slot }}, L_{\text {slot }}\right)$. As a consequence, the insertion loss of the RDMS unit in the All-off state can be expressed by

$$
L_{u}(\text { All-off })=L_{m}+L_{d r}+F_{1}\left(W_{\text {slot }}, L_{\text {slot }}\right) .
$$

The reflection is attributed to the step in the RDMS unit as shown in Fig. 2. The transmission line model of the two RDMS units is given in Fig. 2(c). The characteristic impedances of the slot area and the microstrip line surrounding the slot are $Z_{1}$ and $Z_{0}$, respectively. The $Y_{L}$ and $Z_{L}$ caused by the loads have minimum effects on the characteristic impedance. Therefore, given the dimensions of $W, P$, and $g_{2}$, it is calculated that $\left\{Z_{1}, Z_{0}\right\}=\{22 \Omega, 45 \Omega\}$ and $\{50 \Omega, 106 \Omega\}$ for the original and the new RDMS units, respectively. It is noticed that due to the width reduction, the new RDMS has larger $Z_{1}$ and $Z_{0}$ compared to those of the previous one, the normalized characteristic impedances $Z_{1} / Z_{0}$ for the two RDMS units are similar. Therefore, the reflection $F_{1}$ can maintain at the same level with the same $L_{\text {slot }}$. This 


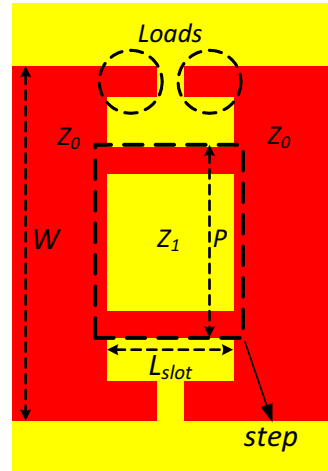

(a)

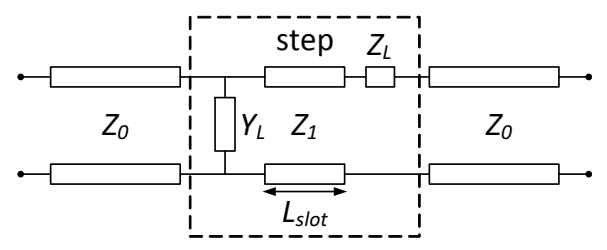

(c)

Fig. 2. Equivalent structure of (a) the original RDMS unit and (b) the new RDMS unit in the All-off state, and (c) their transmission line model.

explains why the achieved width reduction does not increase the insertion loss.

\section{B. Variation law}

It is found in equations (2) and (3) that the insertion loss can be seen as a constant in the All-on state, and a function of $W_{\text {slot }}$ and $L_{\text {slot }}$ in the All-off state, respectively. The phase shift is also a function of the slot dimensions and can be expressed by

$$
\theta=F_{2}\left(W_{\text {slot }}, L_{\text {slot }}\right),
$$

since the variable $t$ in equation (1) is a linear function of $W_{\text {slot }}$ $\left(W_{\text {slot }}=2 t+g_{2}\right)$. To demonstrate the performance of the new RDMS unit shown in Fig. 1(b) relative to the slot dimensions, parametric studies on $W_{\text {slot }}$ and $L_{\text {slot }}$ are conducted using CST Microwave Studio [23]. In the simulation, the diode is modelled as a $4-\Omega$ resistor in the All-on state, and a parallel circuit consisting of a $20 \mathrm{~K}-\Omega$ resistor and a $0.04-\mathrm{pF}$ capacitor in the All-off state, according to the datasheet [24].

Fig. 3(a) and 3(b) gives the simulated insertion losses and phase shift of the RDMS unit at $5.2 \mathrm{GHz}$, respectively. The insertion loss is defined as $-\left|S_{21}\right|$, and the phase shift is defined as the phase difference between the $S_{21}$ in the All-on state and other states. It is observed in Fig. 3(a) that the RDMS unit in the All-on state has a low insertion loss that remains unchanged with different $W_{\text {slot }}$ and $L_{\text {slot }}$, which is expected from equation (2). However, the All-off state insertion loss increases with the slot dimensions. As shown in Fig. 3(b), there is a soar in the phase shift of the RDMS unit by increasing $W_{\text {slot }}$ and $L_{\text {slot }}$. It is concluded from the results that both the

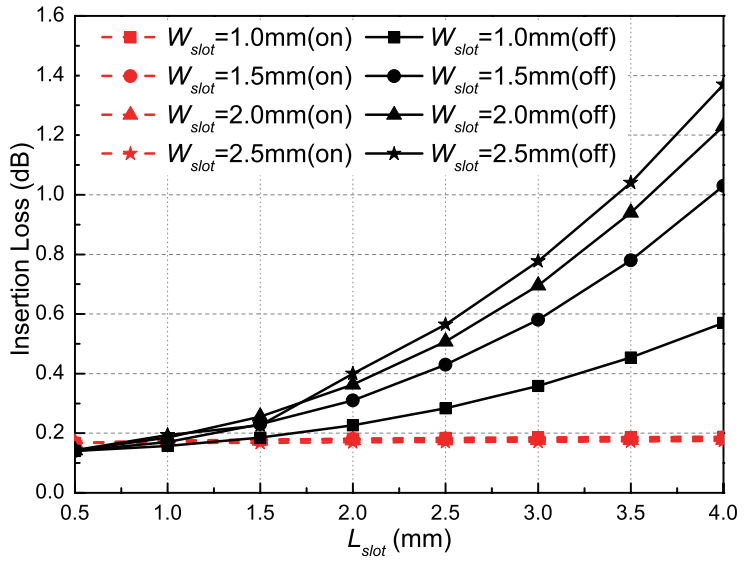

(a)

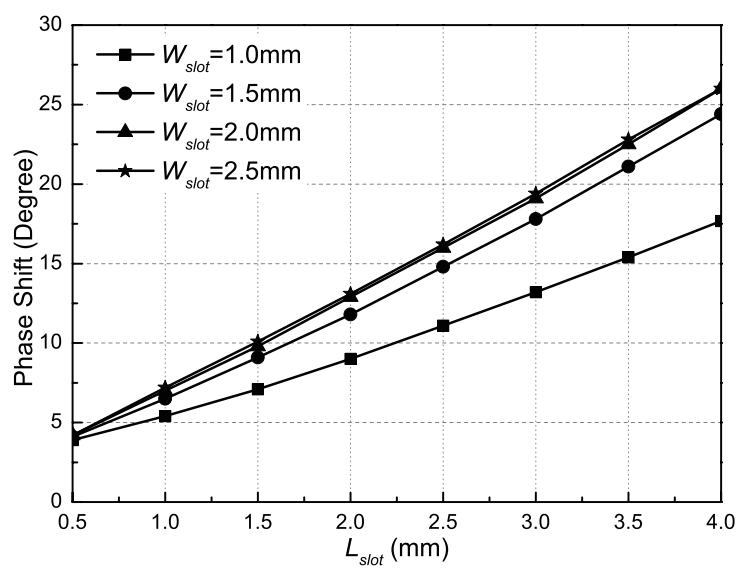

(b)

Fig. 3. Simulated (a) insertion losses and (b) phase shifts of the RDMS unit with different slot dimensions.

$F_{1}$ and $F_{2}$ in equations (3) and (4) are monotonic increasing functions of $W_{\text {slot }}$ and $L_{\text {slot }}$. Theoretically, any phase shift value can be obtained using a single RDMS unit. However, a higher phase shift is accompanied by a higher insertion loss in the All-off state.

\section{Fabricated RDMS units}

To verify the design concept, four RDMS units have been designed with $\left\{W_{\text {slot }}, L_{\text {slot }}\right\}=\{1 \mathrm{~mm}, 1 \mathrm{~mm}\},\{2 \mathrm{~mm}$, $2.4 \mathrm{~mm}\},\{2 \mathrm{~mm}, 3.3 \mathrm{~mm}\}$, and $\{2 \mathrm{~mm}, 6.2 \mathrm{~mm}\}$ to achieve phase shifts of $\theta=5^{\circ}, 15^{\circ}, 22.5^{\circ}$, and $45^{\circ}$, respectively, at $5.2 \mathrm{GHz}$. The four RDMS units are correspondingly defined as RDMS $_{i}$, where $i=1,2,3$ and 4, respectively. Fig. 4 presents the photo of the RDMS units and a microstrip line with the same length for comparison. It is noted that the microstrip line surrounding the RDMS unit is only for connection and its length is immaterial. As a consequence, the effective length of the RDMS unit is considered as $L_{\text {slot }}$ rather than the length of the microstrip line. All the RDMS units are fabricated on $50 \mathrm{~mm}$ long microstrip lines for the ease of measurement 


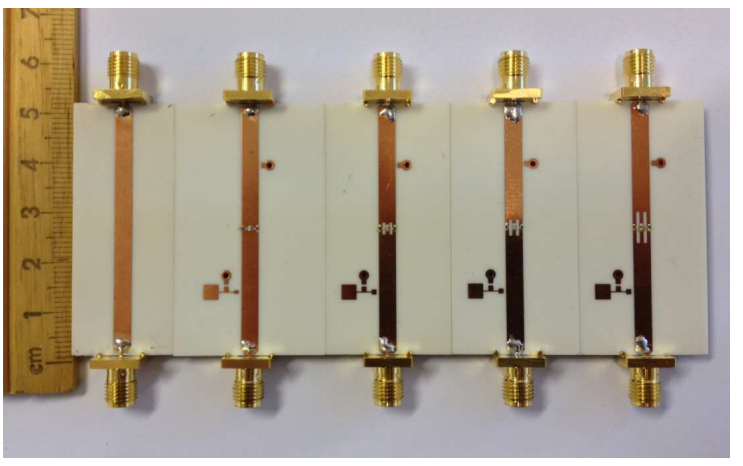

Fig. 4. Photo of the fabricated RDMS units. They are (from left to right) the $50 \mathrm{~mm}$ long uniform microstrip line, $\mathrm{RDMS}_{1}, \mathrm{RDMS}_{2}, \mathrm{RDMS}_{3}$, and $\mathrm{RDMS}_{4}$ units, respectively.

and comparison. It should be pointed out that the RDMS unit working in the All-off state does not need a bias voltage. For the RDMS unit working in the All-on state, a bias voltage of $0.9 \mathrm{~V}$ with the current of $40 \mathrm{~mA}$ is required to keep the two PIN diodes "on".

Fig. 5(a) to 5(c) shows the All-on state insertion losses, Alloff state insertion losses, and phase shifts of the fabricated RDMS units, respectively. The measured insertion loss of the $50 \mathrm{~mm}$ long microstrip line is also presented in Fig. 5(a) as a reference. It is observed from Fig. 5(a) that the measured loss of a microstrip line $L_{m} \approx 0.5 \mathrm{~dB}$, which is mainly attributed to the dielectric loss and SMA losses. Since all the All-on state RDMS units have similar low insertion losses as a uniform microstrip line $\left(L_{u}(\right.$ All-on $\left.)=L_{m}+L_{d r} \approx L_{m}\right)$, it is concluded that the sum of the diode loss and radiation loss $L_{d r} \ll L_{m}$. As shown in Fig. 5(b), the insertion loss in the Alloff state increases with the slot dimensions and can be very high when a large size slot is employed for a higher phase shift, which is in accordance with Fig. 3(a). The measured All-off state insertion losses are 0.5, 0.7, 1.1, and $4.5 \mathrm{~dB}$ for the four RDMS units, respectively. Fig. 5(c) shows that the fabricated RDMS units can achieve phase shifts of $\theta=6^{\circ}$, $15^{\circ}, 20^{\circ}$, and $47^{\circ}$ at $5.2 \mathrm{GHz}$, respectively. This validates the fact that various phase shifts can be obtained by varying $W_{\text {slot }}$ and $L_{\text {slot }}$.

The proposed RDMS unit can serve as a good phase-shifting unit to obtain a phase shift $\theta \leq 15^{\circ}$. However, to obtain a higher phase shift $\theta>15^{\circ}$, the RDMS unit can introduce remarkably high insertion loss in the All-off state. This can be mitigated by using the cascaded structure described in the next section.

\section{RDMS GROUP MADE BY CASCADING RDMS UNITS}

In this section, RDMS groups are produced by cascading several RDMS units described in the previous section. Numerical results show that phase shift introduced by the RDMS group of exactly $\phi=n \theta$ can be achieved, where $\theta$ and $n$ are the phase shift of each RDMS unit and the number of the cascaded units, respectively. Meanwhile, the insertion loss is substantially reduced compared to a single RDMS unit.

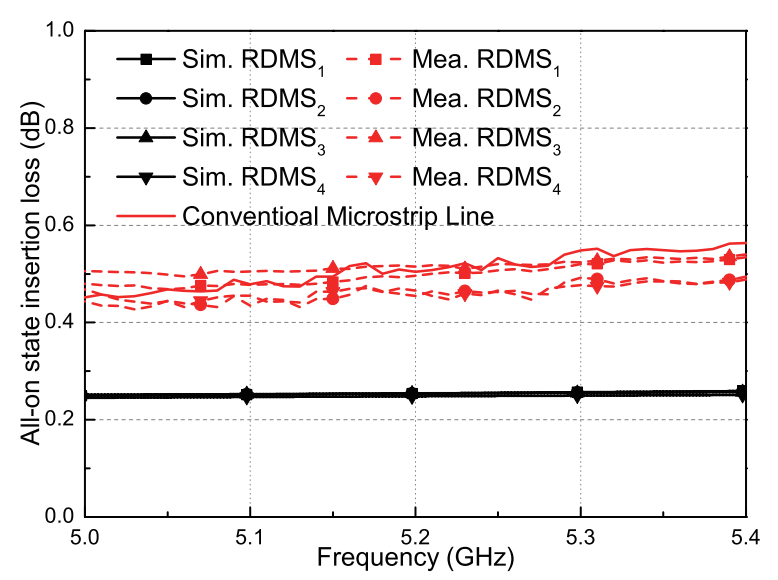

(a)

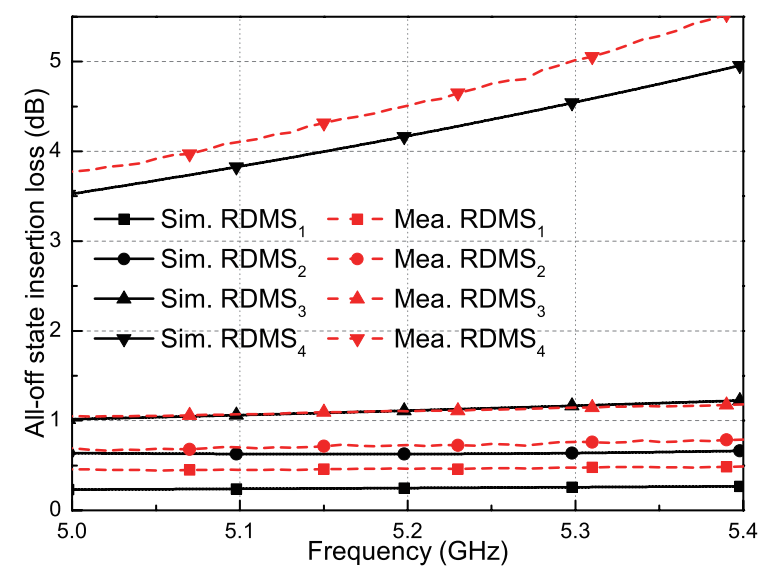

(b)

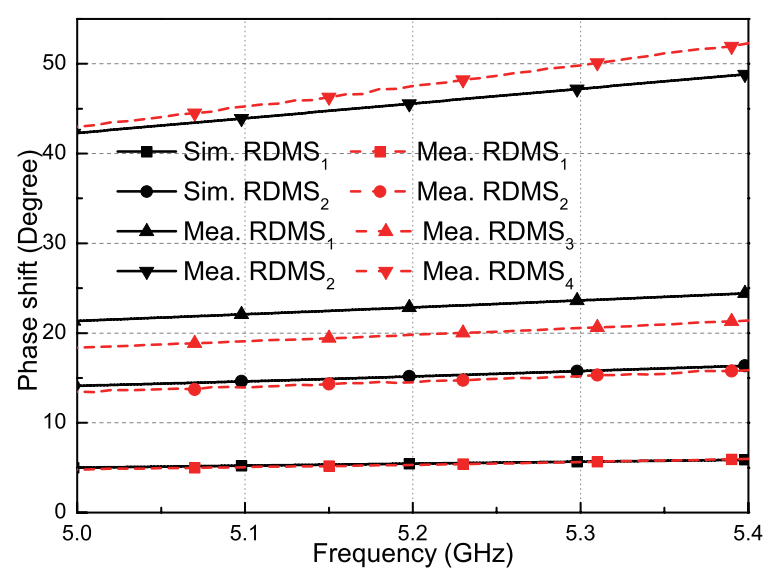

(c)

Fig. 5. Simulated and measured (a) All-on state insertion loss, (b) All-off state insertion loss, and (c) phase shift of the fabricated RDMS units.

\section{A. Cascading analysis}

To illustrate the change of the insertion loss and phase shift when RDMS units are cascaded, a RDMS group consisting of 


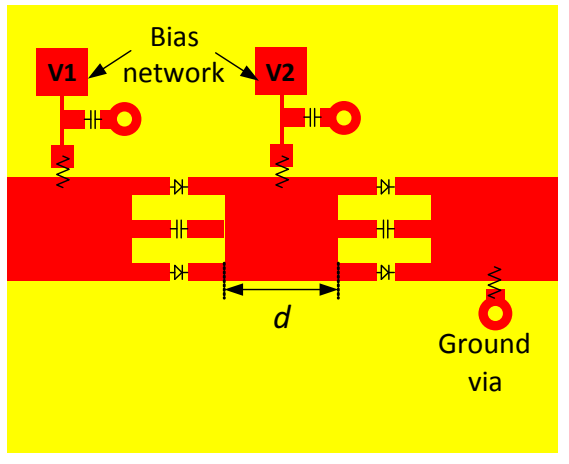

Fig. 6. Structure of the cascaded RDMS units.

two $\mathrm{RDMS}_{3}$ units is proposed as shown in Fig. 6. The two RDMS units are separated from each other by a distance of $d$. Two DC voltages are used to control the two RDMS units independently. As a result, the RDMS group has four working states named as "All-on", "On-off", "Off-on" and "All-off" states when the two RDMS units work in the "All-on, Allon", "All-on, All-off", "All-off, All-on" and "All-off, All-off" states, respectively. It is found that the "On-off" and "Off-on" states have almost the same performance due to the symmetric structure. Therefore, we only present the results of one of them and define it as the "Half-on" state.

The performance (insertion loss and phase shift) of a RDMS group is not only affected by the performance of the RDMS unit employed and the number of the RDMS units cascaded, but also the mutual interaction between the two units, which is related to the distance $d$. A parameter sweep of $d$ from 1 $\mathrm{mm}$ to $4 \mathrm{~mm}$ is conducted using CST microwave studio to study the relationships between the mutual interaction and the performance of the RDMS group. Larger distance $\mathrm{d}>4 \mathrm{~mm}$ are not considered since this will increase the size of the phase shifter too much.

Fig. 7(a) and 7(b) shows the computed insertion losses and phase shifts of the RDMS group, respectively, with different $d$. As shown in Fig. 7(a), the insertion losses in the All-on and Half-on states are independent of $d$. This is due to the fact that a RDMS unit in the All-on state can be simply seen as a uniform microstrip line. Consequently, one of the employed All-on state RDMS units in the group can be replaced by a segment of microstrip line, thus the RDMS group in the All-on and Half-on states are equivalent to one RDMS unit working in the All-on and All-off states, respectively. Therefore, the insertion losses in these two states can be expressed by

$$
\begin{gathered}
L_{g}(\text { All-on })=L_{m}+2 L_{d r}, \\
L_{g}(\text { Half-on })=L_{m}+2 L_{d r}+F_{1}\left(W_{\text {slot }}, L_{\text {slot }}\right),
\end{gathered}
$$

respectively. For the RDMS group in the All-off state, the insertion loss changes with $d$ and is always smaller than that in the Half-on state. In other words, compared to that of a single RDMS unit, the All-off state insertion loss is reduced when two RDMS units are cascaded. This is due to the fact that when the RDMS units are connected by a segment of uniform microstrip line, the input impedance of the RDMS group is changed to be closer to that of the input/output port

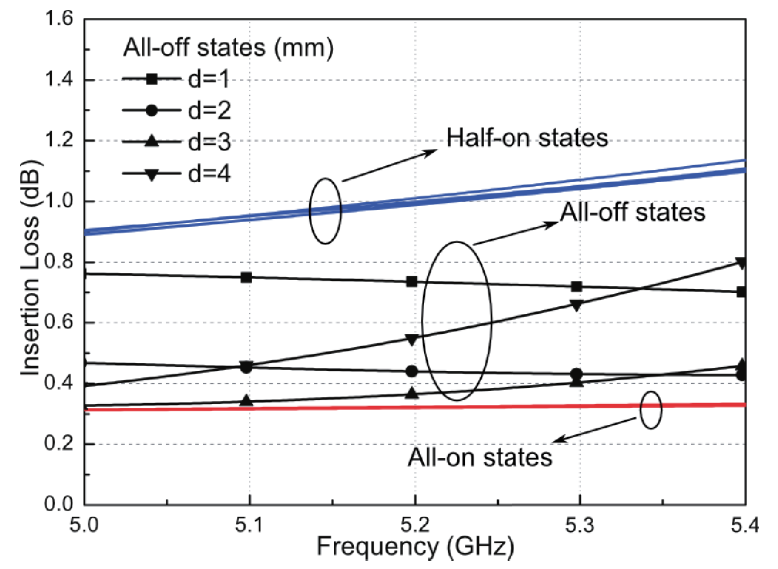

(a)

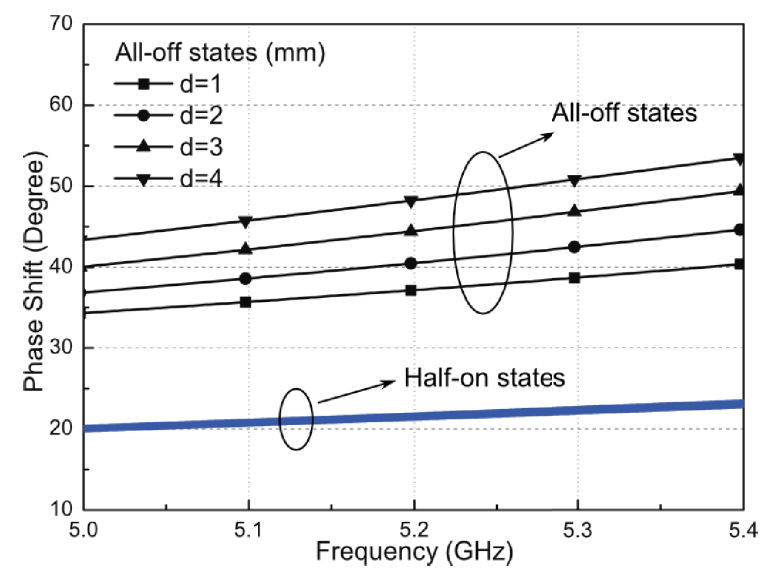

(b)

Fig. 7. Simulated (a) insertion losses and (b) phase shifts of the RDMS group with different $d$.

$(50 \Omega)$, resulting in less reflection. Consequently, the All-off state insertion loss is given as

$$
L_{g}(\text { All-off })=L_{m}+2 L_{d r}+F_{1}\left(W_{\text {slot }}, L_{\text {slot }}\right)-M_{1}(d),
$$

where $M_{1}(d)>0$ represents the reduction in the insertion loss due to the canceled reflection.

The phase shift of the RDMS group in the Half-on state shown in Fig. 7(b) is independent of $d$ and equals to that of a single RDMS unit in the All-off state shown in Fig. 5(c). This is expected and can be attributed to the same reason that we used to explain the insertion loss. With the All-on state phase as a reference, the phase shift in the Half-on state can be expressed as

$$
\phi(\text { Half-on })=F_{2}\left(W_{\text {slot }}, L_{\text {slot }}\right)=\theta .
$$

In the All-off state, the phase shift of the RDMS group is doubled since the current path change is doubled compared to that of a single RDMS unit. In addition, as observed in Fig. 7(b), the phase shift is also affected by the reflection change. 


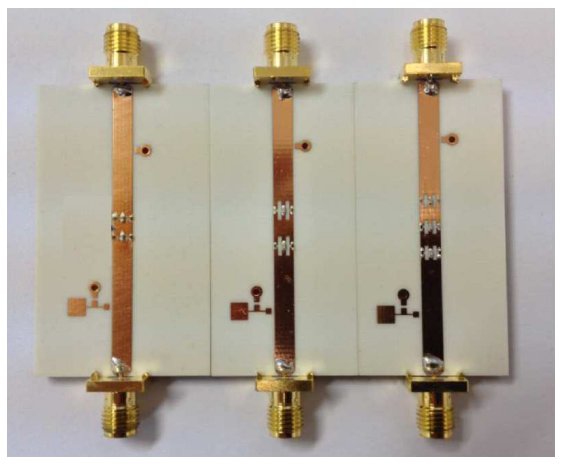

Fig. 8. Photo of the fabricated ${ }^{2} \mathrm{RDMS}_{1},{ }^{2} \mathrm{RDMS}_{3}$, and ${ }^{3} \mathrm{RDMS}_{2}$ groups (from left to right).

Therefore, the phase shift can be expressed by

$$
\phi(\text { All-off })=2 F_{2}\left(W_{\text {slot }}, L_{\text {slot }}\right)+M_{2}(d),
$$

where $M_{2}(d)$ is the phase variation with respect to the reflection change and can be either positive or negative with different $d$. For a specific RDMS group, we can always find a $d$ to satisfy

$$
\left|M_{2}(d)\right|=\text { minimum, }
$$

and

$$
M_{1}(d)>0,
$$

thereby the RDMS group can have an exactly doubled phase shift of $\phi=2 \theta$ and a smaller insertion loss $L_{g}$ (All-off) $<$ $L_{u}$ (All-off) concurrently, compared to those of the employed RDMS unit.

As shown in Fig. 7(a), it is noted that the insertion loss in the Half-on state is obviously larger than those in the Allon and All-off states. As a consequence, we choose to bias the two RDMS units simultaneously rather than control them separately. This way, only one bias network is required and the RDMS group works in two discrete states, the All-on and All-off states.

Moreover, we could also cascade more than two RDMS units to produce a RDMS group. The equations (5), (7), and (9) presenting the insertion losses and phase shift of a RDMS group cascading 2 RDMS units can also be applied to a RDMS group cascading $n$ RDMS units. The expressions of the insertion losses and phase shift can be written as

$$
\begin{gathered}
L_{g}(\text { All-on })=L_{m}+n L_{d r}, \\
L_{g}(\text { All-off })=L_{m}+n L_{d r}+F_{1}\left(W_{\text {slot }}, L_{\text {slot }}\right)-M_{1}(n, d), \\
\phi=n \theta+M_{2}(n, d),
\end{gathered}
$$

where $F_{1}\left(W_{\text {slot }}, L_{\text {slot }}\right)-M_{1}(n, d)$ in (13) and $M_{2}(n, d)$ in (14) could be very small with an optimized $d$.

\section{B. Measurement results of RDMS groups}

For simplicity, a RDMS group cascading $n \mathrm{RDMS}_{i}$ units is defined as ${ }^{n} \mathrm{RDMS}_{i}$ group, where $i$ represents the serial number of the RDMS unit proposed in the previous section, and $n$ is the quantity of the cascaded RDMS units. Two RDMS groups ${ }^{2} \mathrm{RDMS}_{1}$ and ${ }^{2} \mathrm{RDMS}_{3}$ are designed with $d=2 \mathrm{~mm}$

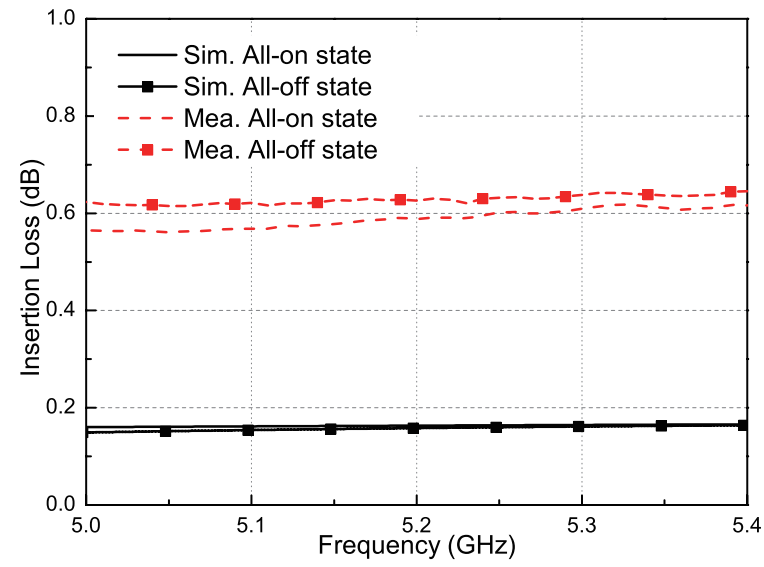

(a)

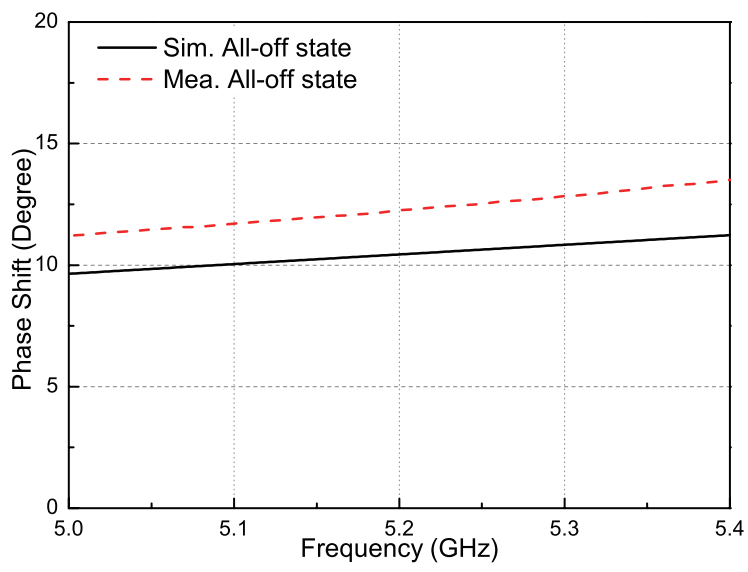

(b)

Fig. 9. Simulated and measured (a) insertion losses and (b) phase shifts of the ${ }^{2} \mathrm{RDMS}_{1}$ group.

and $2.8 \mathrm{~mm}$ to obtain phase shifts of $\phi=2 \theta_{1}$ and $2 \theta_{3}$, respectively, where $\theta_{i}$ is the phase shift of the $\mathrm{RDMS}_{i}$ unit. Also, a RDMS group ${ }^{3} \mathrm{RDMS}_{2}$ is designed with $d=2.4 \mathrm{~mm}$ to achieve a phase shift of $\phi=3 \theta_{2}$. The fabricated RDMS groups are pictured in Fig. 8.To bias a RDMS group cascading $\mathrm{n}$ RDMS units, the required DC voltage and current are $n \times 0.9 \mathrm{~V}$ and $40 \mathrm{~mA}$, respectively.

Figs. 9 to 11 present the simulated and measured results of the ${ }^{2} \mathrm{RDMS}_{1},{ }^{2} \mathrm{RDMS}_{3}$, and ${ }^{3} \mathrm{RDMS}_{2}$ groups, respectively. It is seen that the measured results agree well with the simulated ones. As shown in the figures, the insertion losses for these RDMS groups are all $<1 \mathrm{~dB}$ in both of the two states. Regarding the phase shift, the measured results shown in Figs. 9 and 10 demonstrate that the ${ }^{2} \mathrm{RDMS}_{1}$ and ${ }^{2} \mathrm{RDMS}_{3}$ groups can realize phase shifts of $\phi=12^{\circ}$ and $43^{\circ}$, respectively, which are two times of those realized by the $\operatorname{RDMS}_{1}\left(\theta=6^{\circ}\right)$ and $\mathrm{RDMS}_{3}\left(\theta=20^{\circ}\right)$ units. It is observed in Fig. 11 that the phase shift achieved by the ${ }^{3} \mathrm{RDMS}_{2}$ group is $\phi=45^{\circ}$, which is exactly three times of that of the $\mathrm{RDMS}_{2}$ unit $\theta=15^{\circ}$.

It is concluded from the measure results that a ${ }^{n} \mathrm{RDMS}_{i}$ 


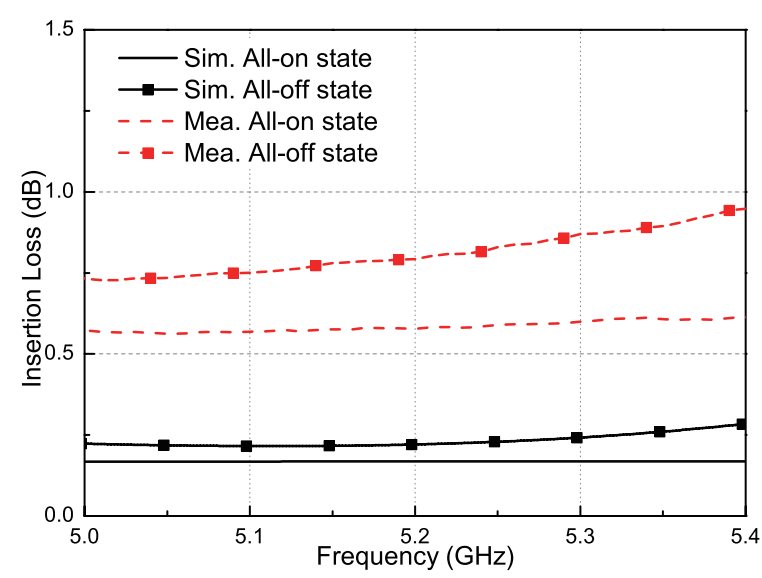

(a)

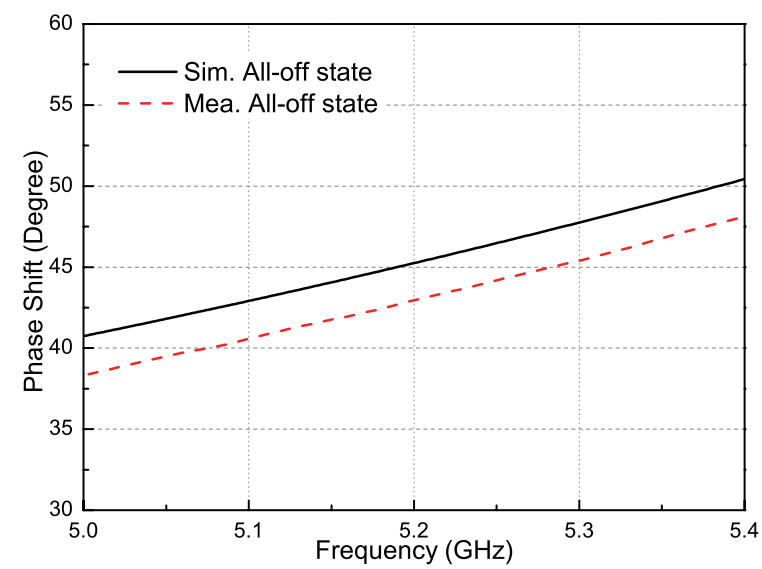

(b)

Fig. 10. Simulated and measured (a) insertion losses and (b) phase shifts of the ${ }^{2} \mathrm{RDMS}_{3}$ group.

group can achieve a phase shift of $\phi=n \theta_{i}$ with low insertion losses, where $\theta_{i}$ is the phase shift of a RDMS ${ }_{i}$ unit. Comparing with the RDMS unit, the RDMS group serves as a better phase shift unit to obtain a phase shift $\phi>15^{\circ}$. For example, to realize a phase shift of $45^{\circ}$, the insertion loss of a RDMS unit is $4.5 \mathrm{~dB}$ while that of a RDMS group is $<0.8 \mathrm{~dB}$.

\section{STEPWISE PHASE SHIFTERS}

\section{A. Design and measurement}

Stepwise phase shifters can be obtained by cascading $m$ RDMS groups and employing more bias networks, where $m$ is a positive integer. This way, a total phase shift of $m \phi$ with a step of $\phi$ can be realized, where $\phi$ is the phase shift of a RDMS group. In order to verify the design scheme, three stepwise phase shifters are designed, fabricated, and tested. The first one is made by cascading two ${ }^{2} \mathrm{RDMS}_{3}$ groups with two bias networks. The second one employs two ${ }^{3} \mathrm{RDMS}_{2}$ groups with two bias networks. Both of these two phase shifters are designed to have a maximum phase shift of $90^{\circ}$ with a step of $45^{\circ}$. The third phase shifter is made by cascading four

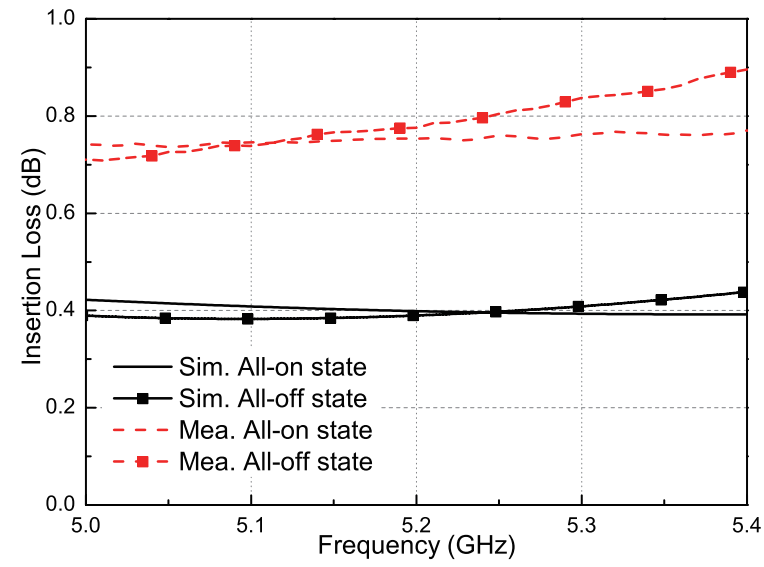

(a)

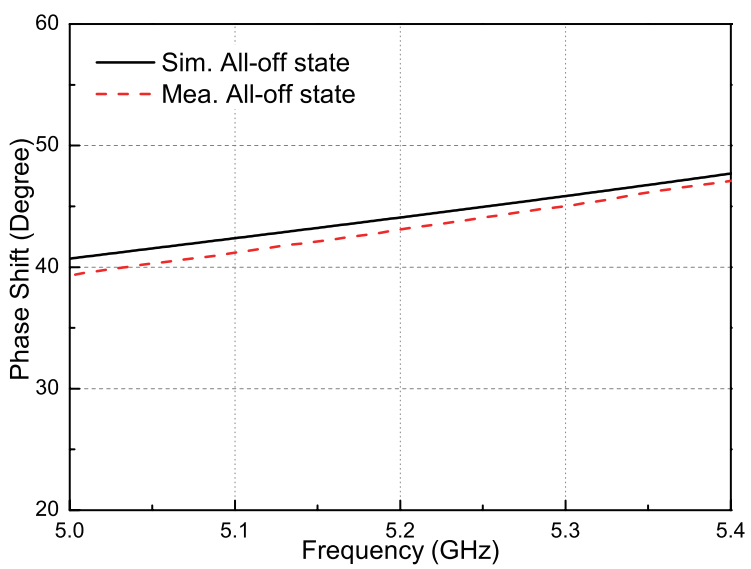

(b)

Fig. 11. Simulated and measured (a) insertion losses and (b) phase shifts of the ${ }^{3} \mathrm{RDMS}_{2}$ group.

${ }^{2} \mathrm{RDMS}_{3}$ groups to obtain a phase shift of $180^{\circ}$. The three phase shifters are named as 4-, 6-, and 8-RDMS phase shifters, respectively. The photo of the fabricated phase shifters are given in Fig. 12.

As shown in Fig. 12, the stepwise phase shifters have employed 2 bias pads for the 4- and 6-RDMS phase shifters and 3 bias pads for the 8-RDMS phase shifters. They are named as Pads 1, 2, and 3, from top to bottom. By manipulating the DC voltages on the bias pads, the stepwise phase shifters are able to work in different states. The 4- and 6-RDMS phase shifters have three states while the 8-RDMS one has five states in terms of phase shift they achieve. The working states, bias voltages, and the ideal phases of the phase shifters are summarized in Table I. The required bias current is always $40 \mathrm{~mA}$ since the RDMS units are connected in series. It should be pointed out that not all the bias pads are connected to a DC source for a certain state. For example, for the All-on state of the 4-RDMS phase shifter, bias pad 2 is not used and marked as "-_" in the table. In addition, for some states of the phase shifters, there is more than one bias method to realize the same phase 


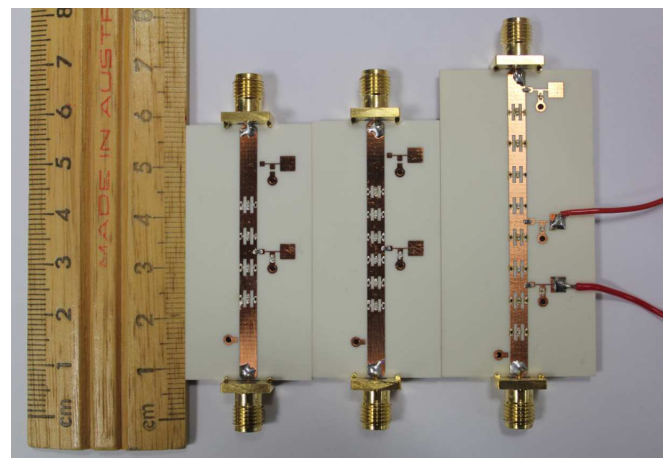

Fig. 12. Photo of the fabricated stepwise phase shifters. They are the 4-, 6-, and 8-RDMS phase shifters from left to right, respectively.

TABLE I

BIAS METHOD OF THE STEPWISE PHASE SHIFTERS AND THEIR IDEAL RELATIVE PHASES OF $S_{21}$ IN DIFFERENT STATES.

\begin{tabular}{|c|c|c|c|c|c|}
\hline $\begin{array}{c}\text { Phase } \\
\text { shifters }\end{array}$ & Pad 1 & Pad 2 & Pad 3 & States name & $\begin{array}{l}\begin{array}{l}\text { Ideal } \\
\text { phase }\end{array} \\
\end{array}$ \\
\hline \multirow{3}{*}{$\begin{array}{l}\text { 4-RDMS } \\
\text { phase shifter }\end{array}$} & $3.6 \mathrm{~V}$ & -- & N/A & All-on & $0^{\circ}$ \\
\hline & $\mathrm{G}$ & $1.8 \mathrm{~V}$ & N/A & Half-on & $-45^{\circ}$ \\
\hline & -- & -- & N/A & All-off & $-90^{\circ}$ \\
\hline \multirow{3}{*}{$\begin{array}{l}\text { 6-RDMS } \\
\text { phase shifter }\end{array}$} & $5.4 \mathrm{~V}$ & -- & N/A & All-on & $0^{\circ}$ \\
\hline & G & $2.7 \mathrm{~V}$ & N/A & Half-on & $-45^{\circ}$ \\
\hline & -- & -- & N/A & All-off & $-90^{\circ}$ \\
\hline \multirow{5}{*}{$\begin{array}{l}\text { 8-RDMS } \\
\text { phase shifter }\end{array}$} & $7.2 \mathrm{~V}$ & -- & -- & All-on & $0^{\circ}$ \\
\hline & $5.4 \mathrm{~V}$ & -- & G & 3-on & $-45^{\circ}$ \\
\hline & $3.6 \mathrm{~V}$ & $\mathrm{G}$ & -- & Half-on & $-90^{\circ}$ \\
\hline & -- & -- & $1.8 \mathrm{~V}$ & 1-on & $-135^{\circ}$ \\
\hline & -- & -- & -- & All-off & $-180^{\circ}$ \\
\hline
\end{tabular}

shift with a similar insertion loss. In this paper, only one way is presented in Table I for the sake of brevity.

Figs. 13 to 15 show the simulated and measured insertion loss and phase shift of the 4-, 6-, and 8-RDMS phase shifters in all the states listed in Table I. It is observed in Fig. 13 that phase shifts of $0^{\circ}, 44^{\circ}$, and $88^{\circ}$ are obtained by the 4RDMS phase shifter in the All-on, Half-on, and All-off states, respectively, at $5.2 \mathrm{GHz}$. The measured insertion losses in all the three states are $<1.3 \mathrm{~dB}$. The 6-RDMS phase shifter introduces phase shifts of $0^{\circ}, 44^{\circ}$, and $87^{\circ}$ in the All-on, Halfon, and All-off states, respectively, as shown in Fig. 14. The insertion losses in all the three states are $<1.2 \mathrm{~dB}$. As shown in Fig. 15, measured phase shifts of $0^{\circ}, 45^{\circ}, 92^{\circ}, 138^{\circ}$, and $184^{\circ}$ are realized by the 8 -RDMS phase shifter. The insertion losses in all the 5 states are $<2 \mathrm{~dB}$ at $5.2 \mathrm{GHz}$.

\section{B. Summarized design scheme of the stepwise phase shifter}

Sections II - IV have described a complete design scheme of the stepwise phase shifter using the proposed RDMS units. To obtain a stepwise phase shifter with a step size of $\phi$ and a total phase-shifting range of $m \phi$ (where $m$ is a positive integer), three steps are required. The first step is to optimize the dimensions of a single RDMS unit to obtain a phase shift of $\theta=\phi / n$ (where $n$ is also a positive integer) at the design frequency. The second step is to obtain a RDMS group with a phase shift of $\phi$ by cascading $n$ RDMS units and optimizing the separation distance. The last step is to cascade $m$ RDMS groups and control each group separately using additional bias networks.

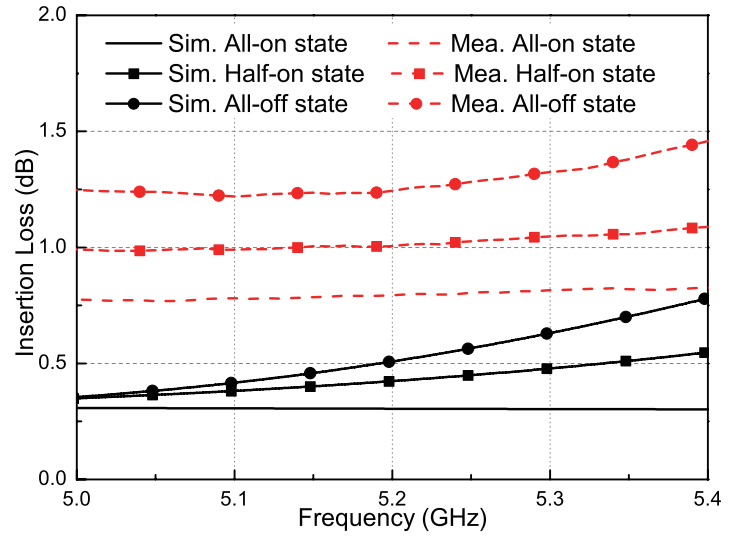

(a)

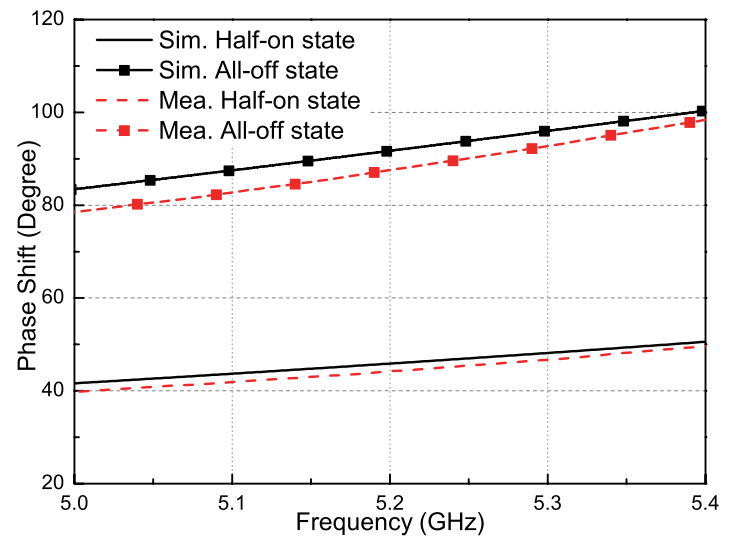

(b)

Fig. 13. Simulated and measured (a) insertion losses and (b) phase shifts of the 4-RDMS phase shifter.

More specifically, some guidelines are described as follows to choose the value of $n$ in the second step depending on the step size $\phi$.

1) Case $1(n=1)$ : To obtain a step size $\phi \leq 15^{\circ}$, a single RDMS unit should be used to realize the step. This is due to the fact that it has the smallest size while the insertion losses are also small as observed in Fig. 5. If using a RDMS group cascading $n$ RDMS units $(n>1)$ to realize such a phase shift, the reduction in insertion loss would be negligible, while the size is noticeably increased.

2) Case $2(n=2)$ : For the step size $15<\phi \leq 50^{\circ}$, a RDMS group cascading 2 RDMS units is the best option, due to the fact that the insertion loss can be fairly high using a single RDMS unit to realize such a phase shift. For example, the measured insertion loss of the $\mathrm{RDMS}_{4}$ unit is $4.5 \mathrm{~dB}$ with a $45^{\circ}$ phase shift. Actually, a similar small insertion loss can also be obtained using RDMS groups cascading more than 2 RDMS units, but this would increase the size.

3) Case $3(n>2)$ : To obtain higher step size $\phi>50^{\circ}$, a RDMS group cascading $n(n>2)$ RDMS units is required. However, it should be borne in mind that the size of the RDMS group increases with $n$. Therefore, if the size rather than the 


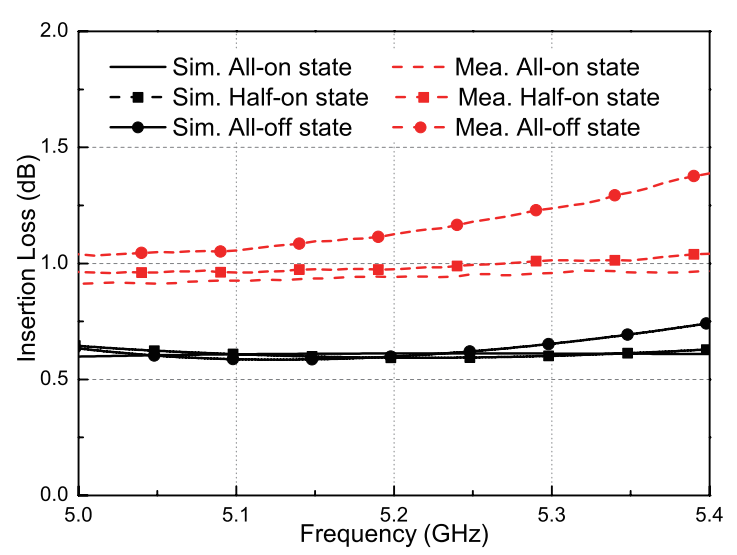

(a)

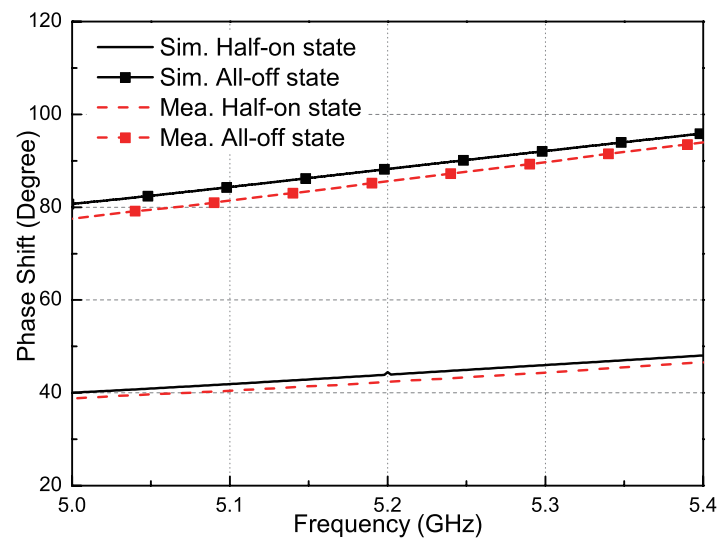

(b)

Fig. 14. Simulated and measured (a) insertion losses and (b) phase shifts of the 6-RDMS phase shifter.

insertion loss is the main concern, a smaller $n$ is preferred.

As the significant improvements to the RDMS phase shifters given in [22], the ones obtained in this work not only inherent all the advantages including large phase-shifting range with flexible step size, low insertion loss, easy control method, easy fabrication process, and simple low cost structure compared to the DGS based phase shifters [17-19], but they possess other noticeable merits as follows. Firstly, the size of the phase shifter has been significantly reduced without affecting the phase shift. Compared with the original phase shifters that can achieve phase shifts of $45^{\circ}$ and $90^{\circ}$, the sizes of the new ones in this paper has been reduced to only $10.6 \%$ and $17.5 \%$, respectively, with unchanged phase shifts. The significant size reduction is due to the smaller size of the proposed RDMS unit and the elimination of the impedance matching part. Secondly, the quantity of the lumped elements employed in the new phase shifters is only half of the original ones. This can be attributed to the new configuration that reduces the number of the capacitors used in a RDMS unit and the quantity of the RDMS units in a RDMS group. Thirdly, the new phase shifters have lower insertion losses. The loss reduction is

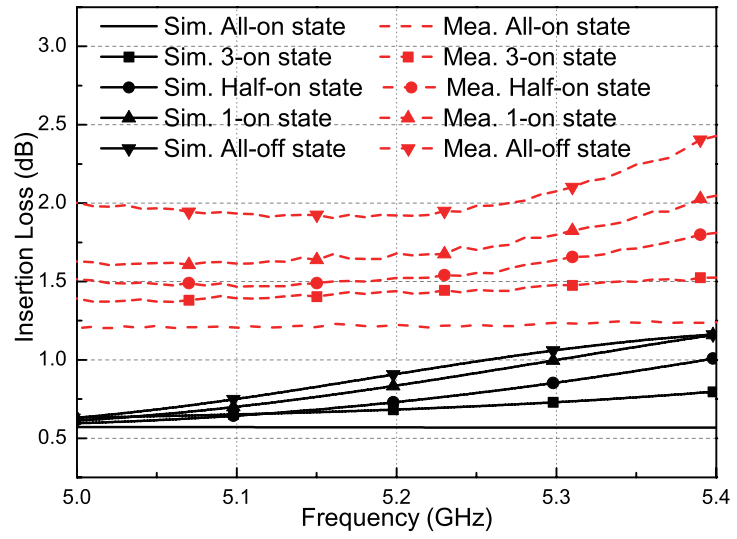

(a)

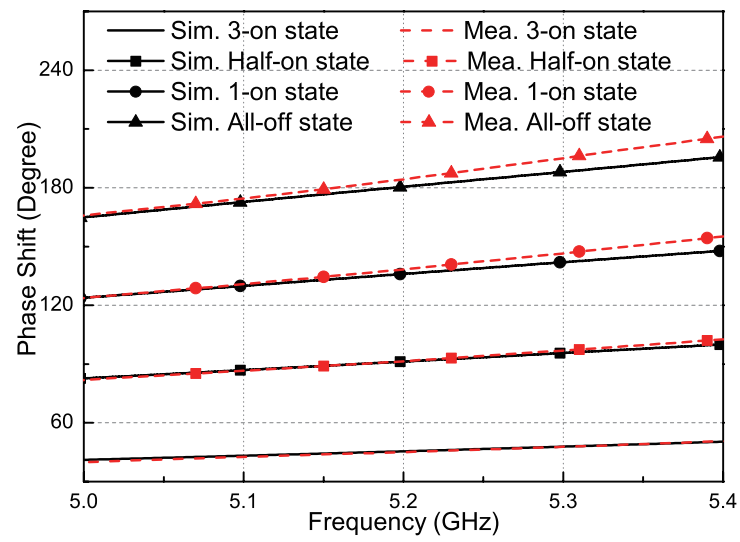

(b)

Fig. 15. Simulated and measured (a) insertion losses and (b) phase shifts of the 8 -RDMS phase shifter.

resulted from a smaller quantity of the lumped elements and a lower dielectric loss due to the smaller size. In addition, unlike the original ones, the new phase shifters do not need tapered microstrip lines for impedance match when connected to a $50-\Omega$ microstrip system, which leads to smaller losses.

\section{A 4-ELEMENT PHASED ARRAY}

The proposed phase shifters are employed in a 4-element phased array feed network as shown in Fig. 16. The array is designed to be able to switch its main beam to $-15^{\circ}, 0^{\circ}$, and $15^{\circ}$ in its H-plane. Meanwhile, the size has been reduced by $55 \%$ compared to the previous work.

In this design, 3 Wilkinson power dividers are employed to split the power while 8 phase shifters are integrated in the feed network to realize $50^{\circ}$ progressive phase differences between the array elements at $5.2 \mathrm{GHz}$. Each phase shifter is composed of 1 RDMS group consisting of two RDMS units with $W_{\text {slot }}=2 \mathrm{~mm}, L_{\text {slot }}=3.6 \mathrm{~mm}$, and $d=3 \mathrm{~mm}$. As shown in Fig. 16, for the first-level power divider, there are 2 phase shifters at each of the 2 branches. For the second-level, there is 1 phase shifter at each of the 4 branches. Two bias 


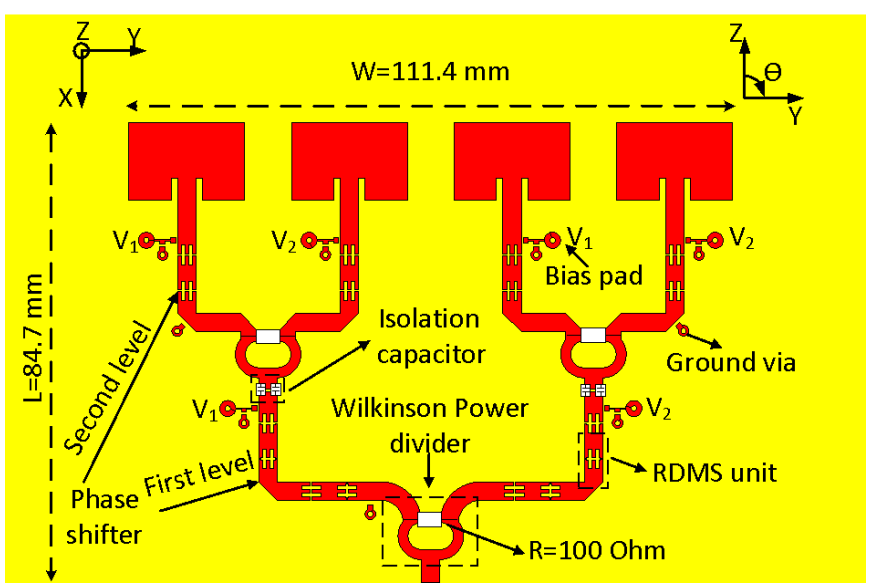

Fig. 16. Prototype of the 4-element phased array.

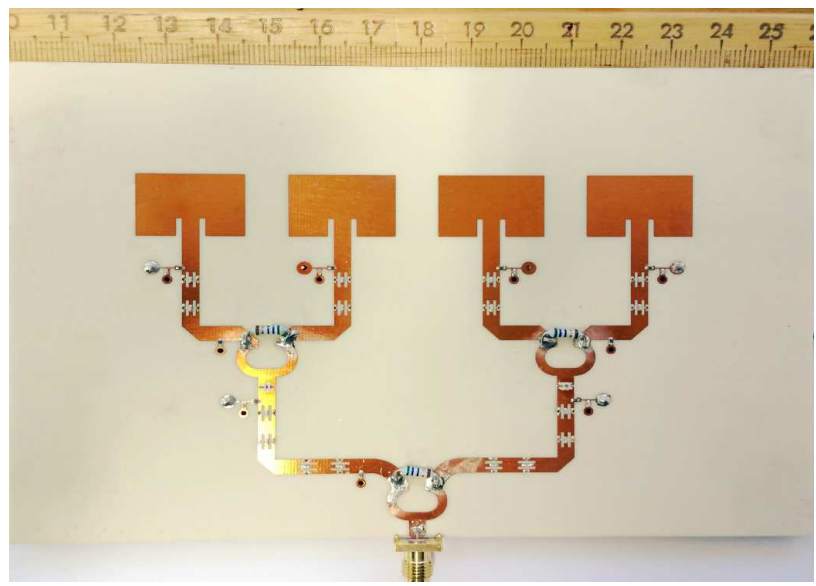

Fig. 17. Photo of the fabricated array.

DC voltages labelled by $V_{1}$ and $V_{2}$ are employed to control the phase shifters. The biasing mechanism is the same as in [22]. There are 4 different working states of the phased array. When voltages $V_{1}$ and $V_{2}$ are "+,+", "-,-", "+,-", and "-,+", the phased array can work in the "All-on", "All-off", "left-onright-off", and "Left-off-right-on" states, respectively. In the All-on and All-off states, the array elements are in phase so that the beam is not tilted. In the left-on-right-off and Leftoff-right-on states, phase advances and delays of $50^{\circ}$ between the array elements are obtained, respectively. Therefore, beam tilts of $-15^{\circ}$ and $15^{\circ}$ are realized.

The 4-element phased array antenna is fabricated and tested. Fig. 17 shows the picture of the fabricated antenna array. The simulated and measured reflection coefficient $S_{11}$ is depict in Fig. 18. According to the figure, the overlapped impedance bandwidth of the 4 states can cover the wireless local area network (WLAN) $5.2 \mathrm{GHz}$ band (e.g. 5.15-5.35 GHz in the USA, 5.15-5.25 GHz in Japan, and 5.15-5.35 GHz in Europe). Fig. 19 shows the simulated and measured co-polarization and cross-polarization patterns in the H-plane (Z-Y plane) of the antenna. As shown in Fig. 19, the phased array can switch its main beam towards $0^{\circ}, 0^{\circ},-15^{\circ}$, and $15^{\circ}$ in the H-plane with minor gain variations for the All-on, All-off, Left-on-rightoff, and Left-off-right-on states, respectively. The measured

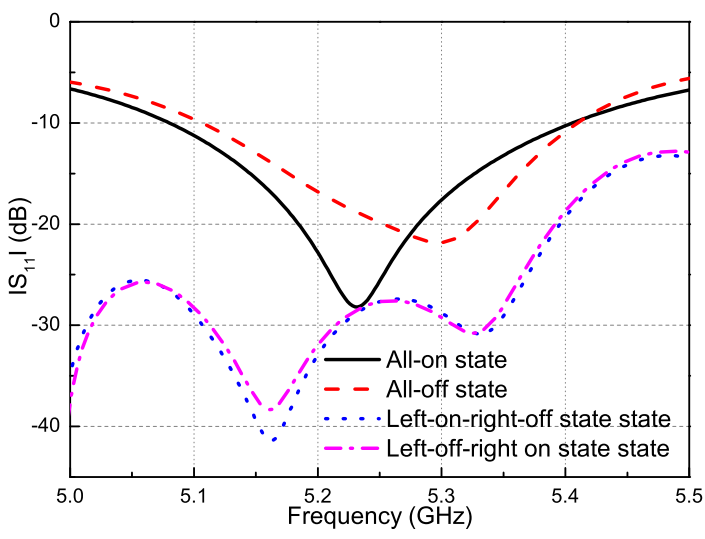

(a)

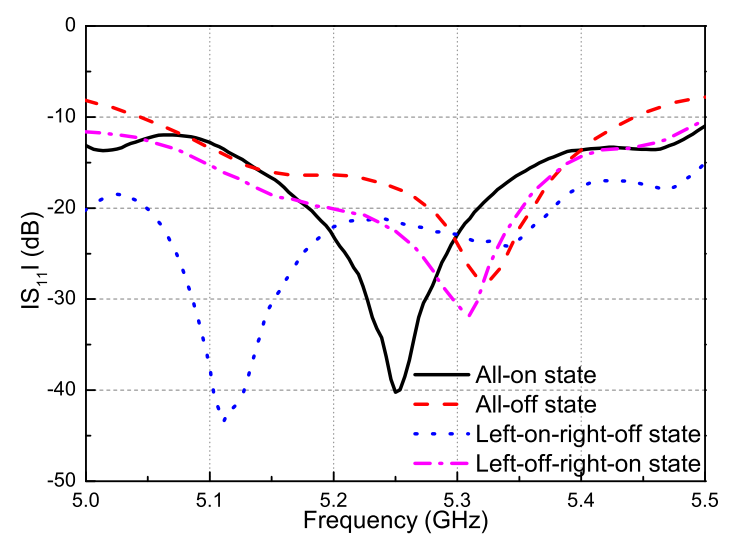

(b)

Fig. 18. (a) Simulated and (b) measured reflection coefficient $S_{11}$ of the phased array.

average gain of the antenna array is $10 \mathrm{dBi}$. The total radiation efficiencies of the antenna in the four states are 78\%, 73\%, $72 \%$, and $72 \%$, respectively.

\section{CONCLUSION}

A new compact reconfigurable defected microstrip structure (RDMS) unit has been proposed. Based on the RDMS units, a complete phase shifter design scheme with three steps has been presented and each step has been elaborated with both theory and measurement. The phase shifters obtained by following such design methodology have significant improvements in size, cost, and loss compared to the designs published before [22]. In addition, significant reductions in cost and size of the array constructed employing such phase shifters are observed while the performance maintains at the same level. The new results reported in this paper demonstrate that the RDMS unit serves as an excellent candidate for phase shifting in microstrip phased antenna arrays. 


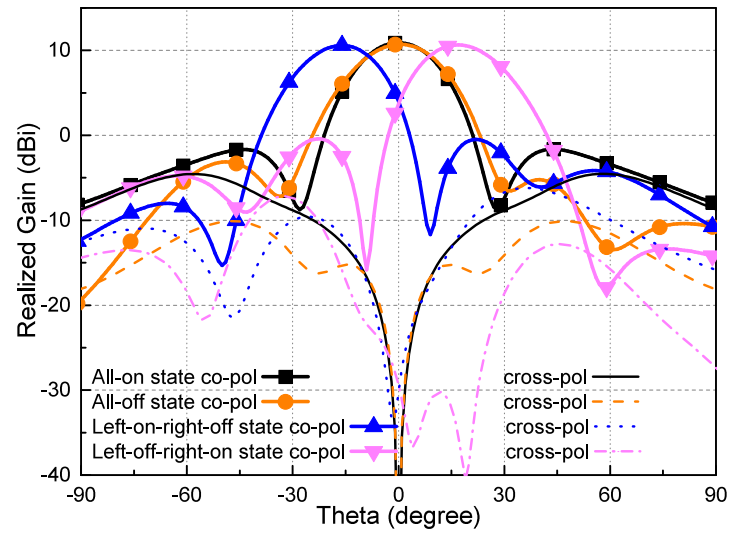

(a)

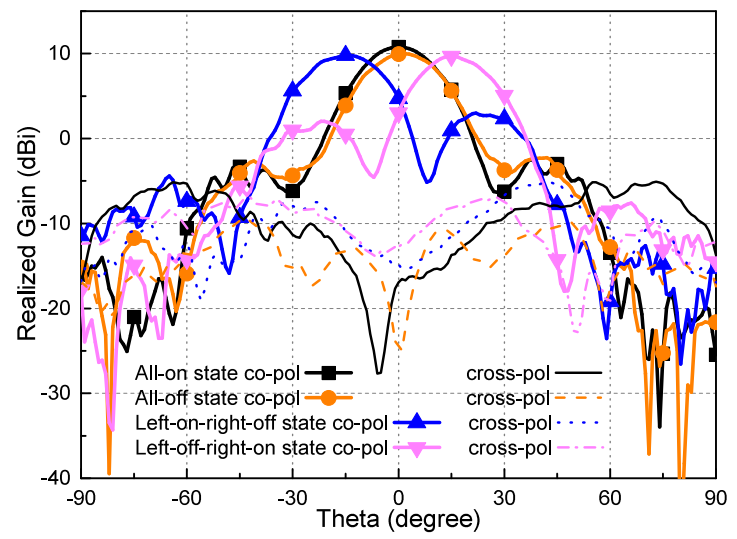

(b)

Fig. 19. (a) Simulated and (b) measured far-field pattern in the H-plane of the phased array.

\section{REFERENCES}

[1] X. J. Huang, Y. J. Guo, and J. D. Bunton, "A hybrid adaptive antenna array," IEEE Trans. Wireless Commun., vol. 9, No. 5, pp. 1770-1779, May 2010.

[2] Y. J. Guo, X. J. Huang, and V. Dyadyuk, "A hybrid adaptive antenna array for long-range mm-wave communications," IEEE Antennas Propagat. Mag., vol. 54, No. 2, pp. 271-282, Apr. 2012.

[3] D. Parker and D. C. Zimmermann, "Phased arrays - part I: theory and architecture," IEEE Trans. Microw. Theory Tech., vol. 50, No. 3, pp. 688-698, Mar. 2002.

[4] R. C. Hansen, Phased array antennas. New York: Wiley, 1998.

[5] J. F. White, "Diode phase shifters for array antennas," IEEE Trans. Microwave Theory Tech. (special issue), vol. 22, pp. 658-674, Jun. 1974.

[6] M. E. Davis, "Integrated diode phase-shifter elements for an X-band phased-array antenna," IEEE Trans. Microwave Theory Tech., vol. 23, pp. 1080-1084, Dec. 1975.

[7] F. G. Terrio, R. J. Stockton, and W. D. Sato, "A low cost p-i-n diode phase shifter for airbone phased-array antenna," IEEE Trans. Microwave Theory Tech. (special issue), vol. 22, pp. 688-692, Jun. 1974.

[8] R. V. Garver, Microwave diode control devices in phase shifters. Norwood, MA: Artech House, 1976, ch. 10.

[9] L. R. Whicker, "Review of ferrite phase shifter technology," IEEE MTT-S Int. Microwave Symp. Dig., pp. 95-97, 1973.

[10] W. E. Hord, C. R. Boyd, Jr., and D. Diaz, "A new type of fast switching dual-mode ferrite phase shifter," IEEE MTT-S Int. Microwave Symp. Dig., vol. 2, pp. 985-988, Jun. 1987.

[11] W. E. Hord, C. R. Boyd, Jr., and D. Diaz, "An accurate anolog ferrite phase shifter," IEEE MTT-S Int. Microwave Symp. Dig., vol. 2, pp. 104 105, May 1971.

[12] D. Parker and D. C. Zimmermann, "Phased arrays - part II: implementations, applications, and future trends," IEEE Trans. Microw. Theory Tech., vol. 50, No. 3, pp. 688-698, Mar. 2002.

[13] R. J. Mailloux, Phased array antenna handbook. Boston, MA: Artech House, 2005.

[14] D. Ahn, J. S. Park, C. S. Kim, J. Kim, Y. Qian, and T. Itoh, "A design of the low-pass filter using the novel microstrip defected ground structure," IEEE Trans. Microw. Theory Tech., vol. 49, no. 1, pp. 86-92, Jan. 2001.

[15] P. Patil, U. P. Khot, and S. Bhujade, "DGS based microstrip phase shifters," in 6th Int. Conf. Sensing Tec. (ICST), Dec. 2012, pp. 723-728.

[16] S. M. Han, C. -S. Kim, D. Ahn and T. Itoh, "Phase shifter with high phase shifts using defected ground structures," Electron. Lett., vol. 41, no. 4, pp. 196-197, Feb. 2005.

[17] C. Shafai, S. K. Sharma, L. Shafai, and D. D. Chrusch, "Microstrip phase shifter using ground-plane reconfiguration," IEEE Trans. Microw. Theory Tech., vol. 52, no. 1, pp.144-153, Jan. 2004.

[18] C. Shafai, S. K. Sharma, J. Yip, and L. Shafai, "Microstrip delay line phase shifter by actuating integrated ground plane membranes," IET Microw. Antennas Propag., vol. 2, no. 2, pp. 163-170, Mar. 2008.

[19] S. Hage-Ali, "A millimeter-wave elastomeric microstrip phase shifter," in IEEE MTT-S international Microwave Symposium, Jun. 2012, pp. 1-3.

[20] J. A. Tirado-Mendez, "A proposed defected microstrip structure (DMS) behavior for reducing rectangular patch antenna size," Microw. Opt. Technol. Lett., vol. 43, no. 6, pp. 481-484, Dec. 2004.

[21] S. Ye, X. L. Wang, W. Z. Wang, R. H. Jin, J. P. Geng, T. S. Bird, and Y. J. Guo, "High Gain Planar Antenna Arrays for Mobile Satellite Communications," Antennas Propagat. Mag., vol. 54, no. 6, pp. 256-268, Dec. 2012.

[22] C. Ding, Y. J. Guo, P. Y. Qin, T. S. Bird, and Y. T. Yang, "A defected microstrip structure (DMS) based phase shifter and its application in beamforming antennas" IEEE Trans. Antennas Propag. vol. 62, no. 02, pp. 641-651, Feb. 2014.

[23] Computer Simulation Technology TM, Darmstadt, Germany, CST Studio Suite, 2012.

[24] M/A-COM datasheet for MA4FCP300.

[25] Datasheet of ATC 600L Series Capacitors.

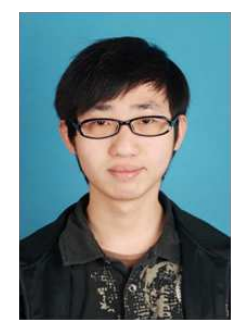

Can Ding was born in Anhui Province, China, in 1989. He received the B. S. degree in Microelectronics from Xidian University, Xi'an, China, in 2009. From 2010 to 2011, he had been working towards the master degree in Micro-electronics from Xidian University and then transferred to Ph. D. He is currently under the cotutelle agreement between Macquarie University and Xidian University. He is also with CSIRO DPaS Flagship, Marsfield, Australia.

His research interest is in the area of reconfigurable antenna, phased array antenna, phase shifter, and microwave circuit design. 


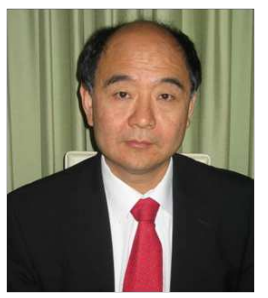

Y. Jay Guo (Fellow2014) received a Bachelor Degree and a Master Degree from Xidian University in 1982 and 1984, respectively, and a PhD Degree from Xian Jiaotong University in 1987, all in China. He has published over 110 journal and 200 refereed international conference papers in antennas, signal processing and wireless communications. $\mathrm{He}$ is a Fellow of the Australian Academy of Technological Sciences and Engineering (ATSE), a Fellow of IEEE and a Fellow of IET.

Jay serves as a Distinguished Professor and the Director of Global Big Data Technologies Centre at the University of Technology Sydney (UTS), Australia. Prior to this appointment, in 2014, he was with CSIRO for over nine years, directing a number of research programs including Smart and Secure Infrastructure, Broadband Networks and Services, Broadband for Australia and Safeguarding Australia. Before joining CSIRO, Jay held various senior leadership positions in Fujitsu, Siemens and NEC in the U.K.

Jay is the recipient of 2012 Australian Government Engineering Innovation Award, 2007 Australian Engineering Excellence Award, 2007 and 2012 CSIRO Chairman's Medal and 2012 CSIRO Newton Turner Award. He has published three technical books, 93 journal papers and 160 refereed international conference papers, and holds 18 patents. He is an Adjunct Professor at University of New South Wales, Macquarie University, University of Canberra, all in Australia, and a Guest Professor at the Chinese Academy of Science (CAS) and Shanghai Jiaotong University.

Jay has chaired numerous international conferences. He is International Advisory Committee Chair of IEEE VTC2017, General Chair of ISAP2015, iWAT2014 and WPMC'2014, Patronage and Publicity Chair of IEEE ICC2014, TPC Chair of 2010 IEEE WCNC, and TPC Chair of 2007 and 2012 IEEE ISCIT. He serves as Guest Editor of special issues on Antennas for Satellite Communications and Antennas and Propagation Aspects of 60$90 \mathrm{GHz}$. Wireless Communications, both in IEEE Transactions on Antennas and Propagation, and Special Issue on Communications Challenges and Dynamics for Unmanned Autonomous Vehicles, IEEE Journal on Selected Areas in Communications (JSAC).

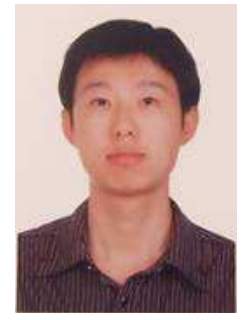

Pei-Yuan Qin (M 13) was born in Liaoning Province, China in 1983. He received a Bachelor Degree in electronic engineering from Xidian University, Xian, China, in 2006, and a joint Ph.D. Degree from Xidian University and Macquarie University, Australia, in electromagnetic fields and microwave technology in 2012 .

From 2012 to 2015, he was a Postdoctoral Research Fellow in Commonwealth Scientific and Industrial Research Organisation (CSIRO), Australia. $\mathrm{He}$ is currently a lecturer with Faculty of Engineering and Information Technology, University of Technology, Sydney (UTS) His research interests are in the areas of reconfigurable antennas, phase shifters, reconfigurable reflectarrays, and MIMO communications.

Pei-Yuan was a recipient of an international Macquarie University research excellence scholarship and was awarded the vice-chancellors commendation for academic excellence by Macquarie University.

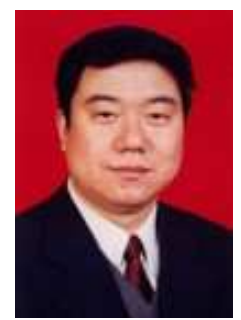

Yintang Yang received the B.S. and M.S. degrees in semiconductor devices and microelectronics from Xidian University, Xi' an, China, in 1982 and 1984, respectively, and the Ph.D. degree in micro- electronics and solid-state electronics from Xi'an Jiaotong University, Xi' an, in 2000

Since 1984, he has been with the Department of Technical Physics at Xidian University. Since 1997, he has been a Professor of Microelectronics and the Director of the Institute of Microelectronics at Xidian University. He has published three books and more than 140 journal and conference papers. His research interests include design methodologies and techniques for analog and mix-signal CMOS integrated circuits, advanced semiconductor materials and devices, as well as micromechanical system and sensors.

Dr. Yang received the National Science Fund for Distinguished Young Scholars from the National Natural Science Foundation of China (NSFC) in 2007. He has been on editorial board for four journals. 